\title{
The Approximate Method for Solving the Boundary Integral Equations of the Problem of Wave Scattering by Superconducting Lattice
}

\author{
Gandel Yu. V. ${ }^{1}$, Dushkin V.D.,* \\ ${ }^{1}$ Department of Mathematical Physics and Computational Mathematics, Karazin Kharkiv National University, Kharkiv, Ukraine \\ ${ }^{2}$ Department of Fundamental Science, National Academy of NGU, Kharkiv, Ukraine \\ *Corresponding author: dushkinvd@gmail.com
}

Received October 18, 2014; Revised November 10, 2014; Accepted November 19, 2014

\begin{abstract}
In this article the method for numerical solution of boundary integral equations of the original problem is proposed. This method is one of the modifications of Nystrom-type methods; particularly the method of discrete vortices. The convergence of the numerical solutions to the exact solution of the problem is guaranteed by propositions proved in this article. Also, the rate of convergence of the approximate solutions to the exact solution had been found.
\end{abstract}

Keywords: singular integral equation, modification of method of discrete vortices, existence of approximate solution, the rate of convergence of the approximate solutions

Cite This Article: Gandel Yu. V., and Dushkin V.D., "The Approximate Method for Solving the Boundary Integral Equations of the Problem of Wave Scattering by Superconducting Lattice.” American Journal of Applied Mathematics and Statistics, vol. 2, no. 6 (2014): 369-375. doi: 10.12691/ajams-2-6-3.

\section{Introduction}

The Nystrom-type methods are popular methods of numerical solution of boundary integral equations of mathematical diffraction theory $[1,2,3,4]$. In the countries of the former Soviet Union, some variants of Nystromtype methods are called methods of discrete vortices [5,6] or methods of discrete singularities [7]. In the articles [1,2,3,4] and many others (see. [8]) the solution of mathematical electrodynamics problems is carried out in two stages.

At the first stage the initial boundary-value problem for the Helmholtz equation is reduced to an equivalent system of boundary integral equations using the method of parametric representation of integral operators $[8,9,10]$. On the second stage the resulting system of integral equations are solved numerically by Nystrom-type methods. Using this approach the electrodynamics' structures containing not perfectly conducting and superconducting elements $[12,13,14,15,16]$ can also be investigated numerically.

In particular, the system of boundary integral equations of the problem of electromagnetic waves scattering by a system of superconducting band had been taken in the article [12].

The scheme for numerical solution of this problem and the results of numerical experiments had been proposed in the article [13]. A rigorous mathematical justification for the numerical solution of various electromagnetic problems by the method of discrete singularities $[6,17,18]$ had existed at the time of publication of the articles $[12,13]$. But the systems of boundary integral equations of the problem of electromagnetic waves scattering by a system of superconducting band are different from the systems of integral equations of other problems that were previously solved numerically by the method of discrete singularities.

Due to these differences rigorous mathematical schemes for the numerical solution of the problem of electromagnetic waves scattering by a system of superconducting band has not been given until now.

In the articles $[1,2,16]$ the integral equations have the same properties as the boundary equations of the problem of wave scattering by superconducting tapes.

Therefore, the rigorous justification of schemes for the numerical solution of the problem of wave scattering by superconducting tapes is of interest.

In this article a scheme for the numerical solution of boundary integral equations of the problem of wave scattering by superconducting lattice has been justified. Also, the rate of convergence of the approximate solutions to the exact solution has been found.

\section{The System of Boundary Integral Equations}

The system of boundary integral equations of the problem of wave scattering by superconducting tape consists of integral equations of two different types.

The Equations of the first type are singular integral equations of the first kind: 


$$
\begin{aligned}
& \frac{1}{\pi} \int_{-1}^{1} \frac{1}{\tau-\xi} \frac{\vartheta(\tau) d \tau}{\sqrt{1-\tau^{2}}}+\frac{1}{\pi} \int_{-1}^{1} Q(\xi, \tau) \frac{\vartheta(\tau) d \tau}{\sqrt{1-\tau^{2}}} \\
& -\frac{C_{1}}{\pi} \int_{-1}^{\xi} \frac{\vartheta(\tau) d \tau}{\sqrt{1-\tau^{2}}}=f(\xi),|\xi|<1 ;
\end{aligned}
$$

with the additional condition

$$
\int_{-1}^{1} \frac{\vartheta(\tau) d \tau}{\sqrt{1-\tau^{2}}}=0 .
$$

The equations of the second type are Fredholm equations of the second kind:

$$
\begin{aligned}
& v(\xi)-\frac{C_{2}}{\pi} \sqrt{1-\xi^{2}} \cdot \int_{-1}^{1} \ln |\tau-\xi| \frac{v(\tau) d \tau}{\sqrt{1-\tau^{2}}} \\
& +\frac{1}{\pi} \int_{-1}^{1} K(\xi, \tau) \frac{v(\tau) d \tau}{\sqrt{1-\tau^{2}}}=g(\xi), \quad|\xi|<1 .
\end{aligned}
$$

In the equations (1), (3) it is assumed that

$$
\begin{gathered}
f(\xi) \in C^{\mu, \gamma}([-1,1]), g(\xi) \in C^{0, \psi}([-1,1]), \\
Q(\xi, \tau) \in C^{\mu, \gamma}([-1,1] \times[-1,1]), \\
\mathrm{K}(\xi, \tau) \in C^{0, \psi}([-1,1] \times[-1,1]), \\
\gamma>0, \quad 0<\psi \leq \frac{1}{2} .
\end{gathered}
$$

Let $L_{2, \alpha}, \quad\left(\alpha= \pm \frac{1}{2}\right)$, are the Hilbert spaces of measurable functions with the inner product

$$
(u, v)_{\alpha}=\int_{-1}^{1} u(\tau) \cdot \bar{v}(\tau) \cdot\left(1-\tau^{2}\right)^{\alpha} d \tau
$$

and norm $\|v\|_{\alpha}=\sqrt{(v, v)_{\alpha}}$. Also we define the subspaces

$$
L_{2, \alpha}^{0}=\left\{u \in L_{2, \alpha} \mid(u, 1)_{\alpha}=0\right\} .
$$

We introduce the operators (see $[8,19])$ :

$$
\begin{aligned}
& \Lambda: L_{2,-\frac{1}{2}} \rightarrow L_{2,-\frac{1}{2}}, \\
& (\Lambda u)(\xi)=\frac{1}{\pi} \int_{-1}^{1} \ln |\tau-\xi| \frac{u(\tau) d \tau}{\sqrt{1-\tau^{2}}}, \quad|\xi|<1 ; \\
& K: L_{2,-\frac{1}{2}} \rightarrow L_{2,-\frac{1}{2}} \text {, } \\
& (K u)(\xi)==\frac{1}{\pi} \int_{-1}^{1} K(\xi, \tau) \frac{u(\tau) d \tau}{\sqrt{1-\tau^{2}}}, \quad|\xi|<1 ; \\
& Q: L_{2,-\frac{1}{2}} \rightarrow L_{2, \frac{1}{2}}, \\
& (Q u)(\xi)=\frac{1}{\pi} \int_{-1}^{1} Q(\xi, \tau) \frac{u(\tau) d \tau}{\sqrt{1-\tau^{2}}}, \quad|\xi|<1 ;
\end{aligned}
$$

$$
\begin{aligned}
& J: L_{2,-\frac{1}{2}} \rightarrow L_{2, \frac{1}{2}},(J u)(\xi)=\frac{1}{\pi} \int_{-1}^{\xi} \frac{V(\tau) d \tau}{\sqrt{1-\tau^{2}}},|\xi|<1 ; \\
& P: L_{2,-\frac{1}{2}} \rightarrow L_{2,-\frac{1}{2}},(\mathrm{P} u)(\xi)=\sqrt{1-\xi^{2}} \cdot u(\xi),|\xi|<1 ;
\end{aligned}
$$

$\Gamma: L_{2,-\frac{1}{2}}^{0} \rightarrow L_{2, \frac{1}{2}},(\Gamma u)(\xi)=\frac{1}{\pi} \int_{-1}^{1} \frac{u(t)}{t-\xi} \frac{d t}{\sqrt{1-t^{2}}},|\xi|<1(13)$

and mappings

$$
\begin{gathered}
A: L_{2,-\frac{1}{2}}^{0} \rightarrow L_{2, \frac{1}{2}}, \quad A=\Gamma-c_{1} J+Q ; \\
B: L_{2,-\frac{1}{2}} \rightarrow L_{2,-\frac{1}{2}}, \quad B=I-C_{2}(P \Lambda)+K .
\end{gathered}
$$

With the preceding notation (8)-(15), equation (1) with the additional condition (2) and equation (3) can be written as

$$
\begin{aligned}
& A \vartheta=f, \\
& B v=g .
\end{aligned}
$$

\section{Proposition 1}

The operator $A: L_{2,-\frac{1}{2}}^{0} \rightarrow L_{2, \frac{1}{2}}$ is invertible and operator $A^{-1}$ is bounded.

\section{Proof of Proposition 1}

The operator A - is the sum of invertible operator $\Gamma: L_{2,-\frac{1}{2}}^{0} \rightarrow L_{2, \frac{1}{2}}$ (see $[8,19]$ ) and a compact operator $-C_{1} J+Q$. Hence, by virtue of Nikolsky criterion (see [20], p.150),

$$
\text { ind }\left(\left.A\right|_{L_{1,-\frac{1}{2}}^{0}} \underset{1, \frac{1}{2}}{ }\right)=0 .
$$

From the uniqueness of the problem solutions (1)-(2) follows that

$$
\operatorname{dim} \operatorname{ker}\left(\left.A\right|_{L_{2,-\frac{1}{2}}^{0}} \rightarrow L, \frac{1}{2}\right)=0 .
$$

Hence,

$$
A\left(L_{1,-\frac{1}{2}}^{0}\right)=L_{1, \frac{1}{2}} .
$$

Therefore operator A is bijective and bounded. So, by the Banach Isomorphism Theorem (see in [20], p.113) the operator A has the bounded inverse.

\section{Proposition 2}

The operator $B: L_{2,-\frac{1}{2}}^{0} \rightarrow L_{2, \frac{1}{2}}$ is invertible and the operator $B^{-1}$ is bounded. 


\section{Proof of Proposition 2}

The operator $P \Lambda$ is compact as the composition of the bounded operator $P$ and compact operator $\Lambda$. This follows from the compactness of the operator $-c_{2}(P \Lambda)+K$ and the Fredholm Theorem (see. [20], p.146), that

$$
\text { ind }\left(\left.B\right|_{L 2,-\frac{1}{2}} \rightarrow L_{2,-\frac{1}{2}}\right)=0 \text {. }
$$

From the uniqueness of boundary integral equation solution (3) follows that

$$
\operatorname{dim} \operatorname{ker}\left(\left.B\right|_{L 2,-\frac{1}{2}} \rightarrow L_{2,-\frac{1}{2}}\right)=0
$$

Hence

$$
B\left(L_{2,-\frac{1}{2}}\right)=L_{2,-\frac{1}{2}} .
$$

Therefore operator B is bijective and bounded. So by the Banach Isomorphism Theorem (see in [20], p.113) the operator B has the bounded inverse.

\section{The Approximate System of Integral Equations and Its Properties}

Let us consider sets of points

$$
\begin{aligned}
& t_{k}^{n}=\cos \left(\frac{2 k-1}{2 n} \pi\right), \quad k=1, \ldots, n \\
& t_{0, j}^{n}=\cos \left(\frac{j}{n} \pi\right), \quad j=1, \ldots, n-1 .
\end{aligned}
$$

Here the points $\left\{t_{k}^{n}\right\}_{k=1}^{n}$ are the zeros of Chebychev polynomials of the first kind $T_{\mathrm{n}}(\tau)$ and the points $\left\{t_{0, j}^{n}\right\}_{j=1}^{n-1}$ are the zeros of Chebychev polynomials of the second kind $U_{\mathrm{n}-1}(\xi)$.

Let us consider the basis polynomials

$$
\begin{gathered}
l_{1, n-1, k}(\tau)=\left[1+2 \sum_{p=1}^{n-1} T_{p}(\tau) \cdot T_{p}\left(t_{k}^{n}\right)\right] \cdot \frac{1}{n}, \quad k=1, \ldots, n ;(20) \\
l_{2, n-2, j}(\xi)=\frac{U_{n-1}(\xi)}{U_{n-1}^{\prime}\left(t_{0, j}^{n}\right)\left(\xi-t_{0, j}^{n}\right)}, \quad j=1, \ldots, n-1 ;(21)
\end{gathered}
$$

which has the properties

$$
\begin{gathered}
l_{1, n-1, k}\left(t_{i}^{n}\right)=\delta_{i, k}, \quad i=1, \ldots, n ; \\
l_{2, n-2, j}\left(t_{0, l}^{n}\right)=\delta_{j l}, \quad l=1, \ldots, n-1 ;
\end{gathered}
$$

where $\delta_{i, k}$ is the Kronecker delta.
We introduce functions:

$$
\begin{gathered}
K_{n}(\xi, \tau)=\sum_{j=1}^{n} \sum_{k=1}^{n} K\left(t_{j}^{n}, t_{k}^{n}\right) \cdot l_{1, n-1, j}(\xi) \cdot l_{1, n-1, k}(\tau) \\
Q_{n}(\xi, \tau)=\sum_{j=1}^{n-1} \cdot \sum_{k=1}^{n} Q\left(t_{0, j}^{n}, t_{k}^{n}\right) \cdot l_{2, n-2, j}(\xi) \cdot l_{1, n-1, k}(\tau) \\
f_{n}(\tau)=\sum_{k=1}^{n} f\left(t_{k}^{n}\right) \cdot l_{1, n-1, k}(\tau) \\
g_{n}(\xi)=\sum_{j=1}^{n-1} g\left(t_{0, j}^{n}\right) \cdot l_{2, n-2, j}(\xi)
\end{gathered}
$$

The functions $f_{n}(\tau)$ are the Lagrange interpolation polynomials of the function $f(\tau)$ with the nodal points $\left\{t_{k}^{n}\right\}_{k=1}^{n}$ and the functions $g_{n}(\xi)$ are the interpolation polynomials of the function $g(\xi)$ with the nodal points $\left\{t_{0, j}^{n}\right\}_{j=1}^{n-1}$. The functions $K_{n}(\xi, \tau)$ are the Lagrange interpolation polynomials of the function $K(\xi, \tau)$ with the nodal points $\left\{t_{k}^{n}\right\}_{k=1}^{n}$, with respect to both variables. The functions $Q_{n}(\xi, \tau)$ are the Lagrange interpolation polynomials of the function $Q(\xi, \tau)$ with the nodal points $\left\{t_{0, j}^{n}\right\}_{j=1}^{n-1}$ with respect to variable $\xi$ and with the nodal points $\left\{t_{k}^{n}\right\}_{k=1}^{n}$ with respect to variable $\tau$.

Let us consider the following system of integral equations:

$$
\begin{gathered}
\frac{1}{\pi} \int_{-1}^{1} \frac{1}{\tau-\xi} \frac{\vartheta_{n}(\tau) d \tau}{\sqrt{1-\tau^{2}}}+\frac{1}{\pi} \int_{-1}^{1} Q_{n}(\xi, \tau) \frac{\vartheta_{n}(\tau) d \tau}{\sqrt{1-\tau^{2}}} \\
-\frac{C_{1}}{\pi} \int_{-1}^{\xi} \frac{\vartheta_{n}(\tau) d \tau}{\sqrt{1-\tau^{2}}}=f_{n}(\xi), \quad|\xi|<1 ; \\
\int_{-1}^{1} \frac{\vartheta_{n}(\tau) d \tau}{\sqrt{1-\tau^{2}}}=0 ;
\end{gathered}
$$

$$
\begin{aligned}
& v_{n}(\xi)+\frac{1}{\pi} \int_{-1}^{1} K_{n}(\xi, \tau) \frac{v_{n}(\tau) d \tau}{\sqrt{1-\tau^{2}}}- \\
& -\frac{C_{2}}{\pi} \sum_{k=1}^{n} \sqrt{1-\left(t_{k}^{n}\right)^{2}} \cdot l_{1, n-1, k}(\xi) \cdot \int_{-1}^{1} \ln \left|\tau-t_{k}^{n}\right| \frac{v_{n}(\tau) d \tau}{\sqrt{1-\tau^{2}}} \\
& =g_{n}(\xi), \quad|\xi|<1
\end{aligned}
$$

The functions $\vartheta_{\mathrm{n}}(\tau)$ and $v_{\mathrm{n}}(\tau)$ are sought in the class of polynomials of degree $n-1$. Subsequently, the reasoning shows the existence of such solutions.

We define subspaces $\Pi_{n, \alpha}$ of spaces $L_{2, \alpha}$. Elements of these subspaces are polynomials of degree $n$. Also we are taking under considerations the spaces

$$
\Pi_{n, \alpha}^{0}=\left\{u \in \Pi_{n, \alpha} \mid(u, 1)_{\alpha}=0\right\} .
$$

We introduce the operators: 


$$
\begin{gathered}
P_{n}: \Pi_{n-1,-\frac{1}{2}} \rightarrow \Pi_{n-1,-\frac{1}{2}}, \\
\left(\mathrm{P}_{n} u\right)(\xi)=\sum_{k=1}^{n} \sqrt{1-\left(t_{k}^{n}\right)^{2}} u\left(t_{k}^{n}\right) \cdot l_{1, n-1, k}(\xi) ; \\
K_{n}: \Pi_{n-1,-\frac{1}{2}} \rightarrow \Pi_{n-1,-\frac{1}{2}}, \\
\left(K_{n} u\right)(\xi)=\frac{1}{\pi} \int_{-1}^{1} K_{n}(\xi, \tau) \frac{u(\tau) d \tau}{\sqrt{1-\tau^{2}}}, \quad|\xi|<1 ; \\
Q_{n}: \Pi_{n-1,-\frac{1}{2}}^{0} \rightarrow \Pi_{n-2, \frac{1}{2}}, \\
\left(Q_{n} u\right)(\xi)=\frac{1}{\pi} \int_{-1}^{1} Q_{n}(\xi, \tau) \frac{u(\tau) d \tau}{\sqrt{1-\tau^{2}}}, \quad|\xi|<1 ; \\
A_{n}: \Pi_{n-1,-\frac{1}{2}}^{0} \rightarrow \Pi_{n-2, \frac{1}{2}}, \\
A_{n}=\Gamma_{-}-c_{1} J+Q_{n} ; \\
B_{n}: \Pi_{n,-\frac{1}{2}} \rightarrow \Pi_{n,-\frac{1}{2}}, \\
B_{n}=I-c_{2}\left(P_{n} \Lambda_{n}\right)+K_{n} .
\end{gathered}
$$

In notation (29)-(33) the problems (26)-(27) and (28) have the form:

$$
\begin{aligned}
& A_{n} \vartheta_{n}=f_{n}, \\
& B_{n} v_{n}=g_{n} .
\end{aligned}
$$

The following estimates hold true $[8,20]$ :

$$
\begin{gathered}
\left\|f-f_{n}\right\|_{2, \frac{1}{2}} \leq \frac{M_{1}}{n^{\mu+\gamma},} \\
\left\|g-g_{n}\right\|_{L,-\frac{1}{2}} \leq \frac{M_{2}}{n^{\psi}}, \\
\left\|Q-Q_{n}\right\|_{L_{2,-\frac{1}{2}} \rightarrow L_{2,-\frac{1}{2}}} \leq \frac{M_{3}}{n^{\mu+\gamma}}, \\
\left\|K-K_{n}\right\|_{L_{2,-\frac{1}{2}} \rightarrow L} \leq \frac{M_{4}}{n^{\psi}} .-\frac{1}{2}
\end{gathered}
$$

These estimates are the consequences of Jackson's Theorems (see Corollary 1 of Th. 2 in [21], p.128). The constants $M_{1}$ and $M_{3}$ depend only upon $\mu$ and $\gamma$. Also the constants $M_{2}$ and $M_{4}$ depend only upon $\psi$.

\section{Proposition 3}

For all natural $\mathrm{n}$ the following inequality holds true

$$
\left\|A-A_{n}\right\|_{\Pi_{n-1,-\frac{1}{2}}^{0} \rightarrow L} \leq \frac{M_{3}}{n^{\mu+\gamma}} .
$$

In (40) constant $\mathrm{M}_{3}$ depends only upon $\mu$ and $\gamma$. Besides,

$$
\left\|A-A_{n}\right\|_{n-1,-\frac{1}{2}}^{0} \rightarrow L, \frac{1}{2} \rightarrow 0, \quad n \rightarrow \infty .
$$

\section{Proof of Proposition 3}

From (38) and equality

$$
\left\|A-A_{n}\right\|_{\Pi_{n-1,-\frac{1}{2}}^{0}} \rightarrow L, 2, \frac{1}{2}=\left\|Q-Q_{n}\right\|_{\Pi_{n-1,-\frac{1}{2}}^{0}} \rightarrow L, \frac{1}{2}
$$

it follow that proposition 3 is valid.

\section{Proposition 4}

For all natural $n$ the following inequality holds true

$$
\left\|B-B_{n}\right\|_{n-1,-\frac{1}{2}} \rightarrow L 2, \frac{1}{2} \leq \frac{M^{*}}{n^{\psi}} .
$$

In (42) $M^{*}$ depends only upon $\psi$. Besides,

$$
\left.\left\|B-B_{n}\right\|_{n-1,-\frac{1}{2}} \rightarrow L 2,-\frac{1}{2}\right) \rightarrow 0, \quad n \rightarrow \infty .
$$

\section{Proof of Proposition 4}

If the function $s(\xi)$ is the polynomial of degree $n$ then

$$
\sqrt{1-\xi^{2}} \cdot s(\xi) \in C^{0, \frac{1}{2}}([-1,1]) .
$$

From that fact and by corollary of Jackson's Theorems [21], p. 128 follow:

$$
\left\|P u-P_{n} u\right\|_{L,-\frac{1}{2}} \leq \frac{M_{5}}{\sqrt{n}}, \quad u \in \Pi_{n-1,-\frac{1}{2}} .
$$

Hence, the estimation (43) shows that the operator

$$
W_{n}: \Pi_{n-1,-\frac{1}{2}} \rightarrow L_{2, \frac{1}{2}}, \quad W_{n}=P-P_{n}
$$

and operator

$$
P_{n}: \Pi_{n-1,-\frac{1}{2}} \rightarrow L_{2, \frac{1}{2}}
$$

are bounded. Furthermore

$$
\left\|P-P_{n}\right\|_{n-1,-\frac{1}{2}} \rightarrow L 2, \frac{1}{2} \leq \frac{M_{5}}{\sqrt{n}}, \quad \forall n \in N .
$$

The following inequality clearly holds

$$
\begin{aligned}
& \left\|B-B_{n}\right\|_{\Pi-1,-\frac{1}{2}} \rightarrow L 2,-\frac{1}{2} \\
& \leq\left|c_{2}\right| \cdot\left\|P-P_{n}\right\|_{\Pi=1,-\frac{1}{2}} \rightarrow L{ }_{2,-\frac{1}{2}} \cdot\|\Lambda\|_{\Pi-1,-\frac{1}{2}} \rightarrow L \\
& +\left\|K-K_{n}\right\|_{n-1,-\frac{1}{2}} \rightarrow L \text { 2,- }-\frac{1}{2} .
\end{aligned}
$$

It is well-known [8], [19], that

$$
\Lambda: T_{0}(\tau) \mapsto(-\ln 2) \cdot T_{0}(\xi),
$$




$$
T_{n}(\tau) \mapsto-\frac{T_{n}(\xi)}{n}, n \in N
$$

It follows from (47)-(48) that

$$
\|\Lambda\|_{2,-\frac{1}{2}} \rightarrow L_{2,-\frac{1}{2}}=1 .
$$

From (39), (45)-(46) and (45) follow that Proposition 4 is valid for

$$
M^{*}=\left|c_{2}\right| \cdot M_{5}+M_{3} .
$$

We introduce the variables:

$$
\begin{aligned}
& M_{6}=\left(M_{1} \cdot\left\|A^{-1}\right\|_{L_{2,-\frac{1}{2}}^{-1}} \rightarrow L^{0} 2, \frac{1}{2}\right)^{\frac{1}{\mu+\gamma}} ; \\
& M_{7}=\left(M^{*} \cdot\left\|B^{-1}\right\|_{L 2,-\frac{1}{2}}^{-1} \rightarrow L 2,-\frac{1}{2}\right)^{\frac{1}{\psi}} \text {; } \\
& M=\max \left(M_{6}, M_{7}\right) .
\end{aligned}
$$

In the monograph [22] you can see the following theorem.

\section{Theorem 1}

Let $X$ and $Y$ be normed linear spaces and let $\tilde{X} \subset X$ and $\tilde{Y} \subset Y$ be finite-dimensional subspaces of the same dimension. We consider two equations.

The equation for exact solution of the problem

$$
A u=f \quad u \in X, \quad f \in Y
$$

and the equation for the approximate solution of the problem

$$
A \tilde{u}=\tilde{f} \quad \tilde{u} \in \tilde{X}, \quad \tilde{f} \in \tilde{Y}
$$

where $A$ and $\tilde{A}$ are the linear operators

$$
A: X \rightarrow Y, \quad \tilde{A}: \tilde{X} \rightarrow \tilde{Y} .
$$

Assume that:

1) the operator $A$ is invertible and the operator

$$
A^{-1}: Y \rightarrow X,
$$

is bounded,

2) the inequality holds

$$
p=\left\|A^{-1}\right\|_{Y \rightarrow X} \cdot\|A-\tilde{A}\|_{\tilde{X} \rightarrow Y}<1 .
$$

Then

1) for any function $\tilde{f} \in \tilde{Y}$ the equation (54) has the unique solution $\tilde{u}^{*} \in \tilde{X}$;

2) let $\tilde{u}^{*} \in X$ be the solution of equation (53) and let

$$
\delta \equiv\|f-\tilde{f}\|_{Y},
$$

then

$$
\left\|u-\tilde{u}^{*}\right\|_{X} \leq\left\|A^{-1}\right\|_{Y \rightarrow X} \cdot(1-p)^{-1} \cdot\left(\delta+p\|f\|_{Y}\right) .
$$

The Propositions 1-4 and Theorem 1 lead us to the following result.

\section{Theorem 2}

For all natural $n>M$ the following statements hold true.

1) The problems (34) and (35) have a unique solution.

2) $\vartheta_{n} \in \Pi_{n-1,-\frac{1}{2}}^{0}, \quad v_{n} \in \Pi_{n-1,-\frac{1}{2}}$.

3) The sequence $\left\{\vartheta_{n}\right\}_{n=M}^{\infty}$ converged to the exact solution of the problem (16) in the norm of space $L_{2,-\frac{1}{2}}$. Moreover,

$$
\left\|\vartheta_{n}-\vartheta\right\|_{L 2, \frac{1}{2}} \leq \frac{M_{8}}{n^{\mu+\gamma}}, \quad \forall n \in N, n>M .
$$

4) The sequence $\left\{v_{n}\right\}_{n=M}^{\infty}$ converged to the exact solution of the problem (17) in the norm of space $L_{2,-\frac{1}{2}}$. Moreover,

$$
\left\|v_{n}-v\right\|_{L 2,-\frac{1}{2}} \leq \frac{M_{9}}{n^{\psi}}, \quad \forall n \in N, n>M .
$$

\section{Proof of Theorem 2}

Let's define numbers:

$$
\begin{aligned}
& p_{1, n}=\left\|A^{-1}\right\|_{L 2,-\frac{1}{2}} \rightarrow L_{2,-\frac{1}{2}}^{0} \cdot\left\|A-A_{n}\right\|_{\Pi^{0}} \underset{n-1,-\frac{1}{2}}{\rightarrow} 2, \frac{1}{2}, \\
& p_{2, n} \equiv\left\|B^{-1}\right\|_{L 2,-\frac{1}{2}} \rightarrow L_{2,-\frac{1}{2}} \cdot\left\|B-B_{n}\right\|_{n-1,-\frac{1}{2}} \rightarrow L 2,-\frac{1}{2}
\end{aligned}
$$

From the Propositions 3-4 and the existence of bounded operators $A^{-1}$ and $B^{-1}$ it follows that

$$
\left(p_{1, n} \leq 1\right) \wedge\left(p_{2, n} \leq 1\right) \quad \forall n \in N, n>M .
$$

Appealing to the Theorem 1 and (63) it concludes the uniqueness and the existence of the solution of the problems (16)-(17).

Also the estimations

$$
\begin{aligned}
& \left\|\vartheta_{n}-\vartheta\right\|_{L 2, \frac{1}{2}} \leq\left\|A^{-1}\right\|_{L 2,-\frac{1}{2}} \rightarrow L_{2,-\frac{1}{2}}^{0} \cdot\left(1-p_{1, n}\right)^{-1} . \\
& \cdot\left(\left\|f_{n}-f\right\|_{L 2, \frac{1}{2}}+p_{1, n}\|f\|_{L 2, \frac{1}{2}}\right) \\
& \left\|v_{n}-v\right\|_{L 2,-\frac{1}{2}} \leq\left\|B^{-1}\right\|_{L 2,-\frac{1}{2}} \rightarrow{ }_{2,-\frac{1}{2}} \cdot\left(1-p_{2, n}\right)^{-1} \\
& \cdot\left(\left\|g_{n}-g\right\|_{2,-\frac{1}{2}}+p_{2, n} \cdot\|g\|_{L 2,-\frac{1}{2}}\right) \text {. }
\end{aligned}
$$


follow from the statements of Theorem 1 and inequalities (63). By using (36), (37), (64), (65) and Propositions 3-4 we complete the proof of Theorem 2.

\section{The Discretization of Approximate System of Integral Equations}

As a result of substitution of functions $\vartheta_{\mathrm{n}}$ and $v_{\mathrm{n}}$ in the left-hand side of equations (26) and (28) we obtain polynomials of degree $n-2$ and, $n-1$ respectively. The right-hand side of equations (26) and (28) are polynomials of the same degrees.

It is well-known that the interpolating polynomial of the degree $n$ for a given set of distinct $n+1$ nods is unique. From that follows the statement of Proposition 5.

\section{Proposition 5}

Let the statements of Theorem 2 hold true. In order that the integral equations (26) - (28) hold true on the set of the continuum $|\xi|<1$ is sufficient that:

1. the integral equation (26) is carried out on a discrete set of points $\left\{t_{0, j}^{n}\right\}_{j=1}^{n-1}$;

2. the integral equation (28) is carried out on a discrete set of points $\left\{t_{k}^{n}\right\}_{k=1}^{n}$;

3. the additional condition (27) is fulfilled.

By the Proposition 5, the problem (34) is equivalent to the system of equation:

$$
\begin{gathered}
\frac{1}{\pi} \int_{-1}^{1} \frac{1}{\tau-t_{0, j}^{n}} \frac{\vartheta_{n}(\tau) d \tau}{\sqrt{1-\tau^{2}}}+\frac{1}{\pi} \int_{-1}^{1} Q_{n}\left(t_{0, j}^{n}, \tau\right) \frac{\vartheta_{n}(\tau) d \tau}{\sqrt{1-\tau^{2}}} \\
-\frac{C_{1}}{\pi} \int_{-1}^{t_{0, j}^{n}} \frac{\vartheta_{n}(\tau) d \tau}{\sqrt{1-\tau^{2}}}=f_{n}\left(t_{0, j}^{n}\right), \quad j=1, \ldots, n-1 ; \\
\int_{-1}^{1} \frac{\vartheta_{n}(\tau) d \tau}{\sqrt{1-\tau^{2}}}=0
\end{gathered}
$$

and the problem (35) is equivalent to the equation

$$
\begin{aligned}
& v_{n}\left(t_{l}^{n}\right)-\frac{C_{2}}{\pi} \sqrt{1-\left(t_{l}^{n}\right)^{2}} \cdot \int_{-1}^{1} \ln \left|\tau-t_{l}^{n}\right| \frac{v_{n}(\tau) d \tau}{\sqrt{1-\tau^{2}}} \\
& +\frac{1}{\pi} \int_{-1}^{1} K_{n}\left(t_{l}^{n}, \tau\right) \frac{v_{n}(\tau) d \tau}{\sqrt{1-\tau^{2}}}=g_{n}\left(t_{l}^{n}\right), \quad l=1, \ldots, n .
\end{aligned}
$$

We take discretization of equations (66)-(68) using the interpolation-type quadrature formulas $[8,19]$ :

$$
\begin{aligned}
& \frac{1}{\pi} \int_{-1}^{1} \frac{\vartheta_{n}(t)}{t-t_{0 j}^{n}} \frac{d t}{\sqrt{1-t^{2}}}=\frac{1}{n} \sum_{k=1}^{n} \frac{\vartheta_{n}\left(t_{k}^{n}\right)}{t_{k}^{n}-t_{0 j}^{n}}, j=1, \ldots, n-1 ;(69) \\
& \frac{1}{\pi} \int_{-1}^{1} Q_{n}(\xi, \tau) \frac{\vartheta_{n}(\tau) d \tau}{\sqrt{1-\tau^{2}}}=\frac{1}{n} \sum_{k=1}^{n} Q_{n}\left(\xi, t_{k}^{n}\right) \vartheta_{n}\left(t_{k}^{n}\right),|\xi|<1 ;(70) \\
& \frac{1}{\pi} \int_{-1}^{\xi} \frac{\vartheta_{n}(\tau) d \tau}{\sqrt{1-\tau^{2}}}=\frac{1}{\pi n} \sum_{k=1}^{n} \vartheta_{n}\left(t_{k}^{n}\right) \cdot \rho_{k}^{n}(\xi), \quad|\xi|<1,
\end{aligned}
$$

$$
\begin{aligned}
& \rho_{k}^{n}(\xi)=\left[\pi-\arccos \xi-2 \sum_{p=1}^{n-1} \frac{T_{p}\left(t_{k}^{n}\right)}{p} U_{p-1}(\xi) \sqrt{1-\xi^{2}}\right] ;(71) \\
& \frac{1}{\pi} \int_{-1}^{1} K_{n}(\xi, \tau) \frac{v_{n}(\tau) d \tau}{\sqrt{1-\tau^{2}}}=\frac{1}{n} \sum_{k=1}^{n} K_{n}\left(\xi, t_{k}^{n}\right) v_{n}\left(t_{k}^{n}\right),|\xi|<1 ; \\
& \frac{1}{\pi} \int_{-1}^{1} \ln |\tau-\xi| \frac{v_{n}(\tau) d \tau}{\sqrt{1-\tau^{2}}} \\
& =-\sum_{k=1}^{n} v_{n}\left(t_{k}^{n}\right)\left[\ln 2+2 \sum_{p=1}^{n-1} T_{p}(\xi) \cdot \frac{T_{p}\left(t_{k}^{n}\right)}{p}\right] \frac{1}{n},|\xi|<1 .
\end{aligned}
$$

These quadrature formulas are exact for polynomials of degree $n-1$.

As a result of discretization the system of linear algebraic equations in the unknowns $\vartheta_{n}\left(t_{k}^{n}\right), \quad(k=1, \ldots, n) \quad$ and $\quad v_{n}\left(t_{l}^{n}\right), \quad(l=1, \ldots, n)$ had been obtained. This system has the form

$$
\begin{gathered}
\frac{1}{n} \sum_{k=1}^{n} a_{j k} \cdot \vartheta_{n}\left(t_{k}^{n}\right)=f_{n}\left(t_{0, j}^{n}\right), \quad(j=1, \ldots, n-1) ; \\
\frac{1}{n} \sum_{k=1}^{n} \vartheta_{n}\left(t_{k}^{n}\right)=0 ; \\
v_{n}\left(t_{l}^{n}\right)+\frac{1}{n} \sum_{k=1}^{n} b_{l k} \cdot v_{n}\left(t_{k}^{n}\right)=g_{n}\left(t_{l}^{n}\right), \quad l=1, \ldots, n ;
\end{gathered}
$$

where

$$
\begin{aligned}
& a_{j k}=\left(t_{k}^{n}-t_{0 j}^{n}\right)^{-1}+Q_{n}\left(t_{0 j}^{n}, t_{k}^{n}\right) \\
& -C_{1}\left[\begin{array}{l}
1-\frac{\arccos \left(t_{0 j}^{n}\right)}{\pi} \\
\left.-2 \sum_{p=1}^{n-1} \frac{T_{p}\left(t_{k}^{n}\right)}{\pi p} U_{p-1}\left(t_{0 j}^{n}\right) \sqrt{1-\left(t_{0 j}^{n}\right)^{2}}\right]
\end{array}\right.
\end{aligned}
$$

$$
\begin{aligned}
& b_{l k}=K\left(t_{l}^{n}, t_{k}^{n}\right)+ \\
& +\frac{C_{2}}{\pi} \sqrt{1-\left(t_{l}^{n}\right)^{2}} \cdot\left[\ln 2+2 \sum_{p=1}^{n-1} T_{p}\left(t_{l}^{n}\right) \cdot \frac{T_{p}\left(t_{k}^{n}\right)}{p}\right] .
\end{aligned}
$$

The existence and the uniqueness of the solution of this linear algebraic equation system is a consequence of the existence and the uniqueness of the solution of system (66)-(68) and its equivalence to the problems (34)-(35).

After solving the system (66)-(68) of linear algebraic equations we obtain the solutions of the problems (34)-(35) by the formulas:

$$
\begin{aligned}
& v_{n}(\tau)=\sum_{k=1}^{n} v_{n}\left(t_{k}^{n}\right) \cdot l_{1, n-1, k}(\tau), \\
& \vartheta_{n}(\tau)=\sum_{k=1}^{n} \vartheta_{n}\left(t_{k}^{n}\right) \cdot l_{1, n-1, k}(\tau) .
\end{aligned}
$$




\section{Conclusions}

In this article the justification of the method of numerical solution of the boundary integral equation system (1)-(3) of the problem of wave scattering by superconducting tape had been done. This method is one of the modifications of method of discrete singularities. The convergence of the approximations to the exact solution is guaranteed by propositions proved in this article. Also, the rate of convergence of the approximate solutions to the exact solution had been found.

\section{List of Abbreviations}

$C^{\mu, \gamma}([-1,1])$ - the Hölder space. It consists of functions defined on $[-1,1]$, which have the properties:

1. the functions have continuous derivatives up to order $\mu$;

2. the $\mu$-th derivatives of the functions are Hölder continuous with exponent $\gamma, \quad(0<\gamma \leq 1)$.

\section{References}

[1] Bulygin, V.S., Benson, T.M., Gandel, Y.V., Nosich, A.I., "Fullwave analysis and optimization of a TARA-like shield-assisted paraboloidal reflector antenna using a nystrom-type method," IEEE Transactions on Antennas and Propagation, 61(10), 49814989, 2013.

[2] Bulygin, V.S., Nosich, A.I., Gandel, Y.V., " Nystrom-type method in three-dimensional electromagnetic diffraction by a finite PEC rotationally symmetric surface," IEEE Transactions on Antennas and Propagation, 60(10), 4710-4718, 2012.

[3] Nosich, A.A., Gandel, Y.V., Magath, T., Altintas, A., " Numerical analysis and synthesis of 2D quasi-optical reflectors and beam waveguides based on an integral-equation approach with Nystrom's discretization," Journal of the Optical Society of America A: Optics and Image Science, and Vision, 24(9), 28312836, September 2007.

[4] Gandel, Y.V., Zaginaylov, G.I., Steshenko, S.A., "Rigorous electrodyanamic analysis of resonator systems of coaxial gyrotrons," Technical Physics, 49(7), 887-894, July 2004.

[5] Belotserkovsky, S.M., Lifanov, I.K., Method of Discrete Vortices, CRC Press, New York,1993, 4-464.

[6] Lifanov, I.K., Singular Integral Equations and Discrete Vortices, VSP, Utrecht, the Netherlands, 1996, 4-475.

[7] Gandel, Yu.V., "The method of discrete singularities in problems of electrodynamics," Vopr. Kibern., Moscow, 166-183, 1986. [in. Russian].

[8] Gandel, Yu.V., Dushkin, V.D., Mathematical models of twodimensional diffraction problems: Singular integral equations and numerical methods of discrete singularities method, Academy of
IT of the MIA of Ukraine, Kharkov, Ukraine, 2012, 4-544. [in. Russian].

[9] Gandel' Yu.V. "Parametric representations of integral and psevdodifferential operators in diffraction problems," in 10th Int. Conf. on Math. Methods in Electromagnetic Theory MMET-2004, (Dnepropetrovsk, Ukraine, Sept. 14-17), Dnepropetrovsk, 2004, 57-62.

[10] Gandel, Yu. V., "Boundary-Value Problems for the Helmholtz Equation and their Discrete Mathematical Models," Journal of Mathematical Sciences, 171(1), 74-88, 2010.

[11] Gandel, Y.V., Dushkin, V.D., "The method of parametric representations of integral and pseudo-differential operators in diffraction problems on electrodynamic structures," in Proceedings of the International Conference Days on Diffraction DD 2012 (28 May-1 June 2012), St. Petersburg, Russian Federation, 2012, 76-81.

[12] Gandel', Yu.V., Kravchenko, V.F., Pustovoit, V.I. "Scattering of electromagnetic waves by a thin superconducting band," Doklady Mathematics, 54(3), 959-961, 1996.

[13] Gandel, Yu.V., Kravchenko, V.F., Morozova, N.N. "Solving the problem of electromagnetic wave diffraction by a superconducting thin stripes grating," Telecommunications and Radio Engineering (English translation of Elektrosvyaz and Radiotekhnika), 56(2), 15-17, 2001.

[14] Gandel', Yu.V., Sidel'nikov, G.L., "The method of integral equations in the third boundary value problem of diffraction on a bounded grating over a flat screen," Differential Equations, 35(9), 1169-1175, Sept. 1999.

[15] Dushkin, V.D., "Application of the singular integral transform method to the solution of the two-dimensional problem of diffraction of electromagnetic waves from a superconducting layer with rectangular waveguide channels," Telecommunications and Radio Engineering (English translation of Elektrosvyaz and Radiotekhnika), 56(2), 78-85, 2001.

[16] Nesvit, K.V. " Discrete mathematical model of diffraction on precantor set of slits in impedance plane and numerical experiment," International Journal of Mathematical Models and Methods in Applied Sciences, 7(11), 897-906, 2013.

[17] Gandel, Yu.V., Polyanskaya, T.S., "Justification of a numerical method for solving systems of singular integral equations in diffraction grating problems," Differential Equations, 39(9), 12951307, September 2003.

[18] Gandel, Yu.V., Eremenko, S.V., Polyanskaya, T.S. Mathematical problems in the method of discrete currents. Justification of the numerical method of discrete singularities of solutions of twodimensional problems of diffraction of electromagnetic waves, Educational aid. Part II, Kharkov State University, Kharkov, Ukraine, 1992. [in Russian]

[19] Gandel, Yu.V. Introduction to methods of evaluation of singular and hypersingular integrals,: Publshed by Karazin Kharkiv National University, Kharkov, Ukraine, 2002, 44-62, [in. Russian].

[20] Kutateladze, S.S., Fundamentals of Functional Analysis, Kluwer Academic Publishers Group, Dordrecht, the Netherlands, 1996, 113-150.

[21] Natanson, I.P., Constructive Function Theory, Volume 1, Frederic Ungar Puplishing Co., New York, 1964, 128.

[22] Gabdulkhaev B.G. The optimal approximation of solutions of linear problems, Kazan. Univ. Publishing, Kazan, 1980, 19, [in. Russian]. 